\title{
The Aschenbach effect: unexpected topology changes in motion of particles and fluids orbiting rapidly rotating Kerr black holes
}

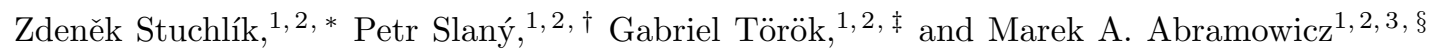 \\ ${ }^{1}$ Institute of Physics, Silesian University at Opava, \\ Bezručovo nám. 13, CZ-746 01 Opava, Czech Republic \\ ${ }^{2}$ NORDITA, Blegdamsvej 17, DK-2100 Copenhagen, Denmark \\ ${ }^{3}$ Theoretical Physics, Göteborg 83 Chalmers Universities, S-412 96 Göteborg, Sweden
}

(Dated: September 18, 2018)

\begin{abstract}
Newton's theory predicts that the velocity $\mathcal{V}$ of free test particles on circular orbits around a spherical gravity center is a decreasing function of the orbital radius $r, \mathrm{~d} \mathcal{V} / \mathrm{d} r<0$. Only very recently, Aschenbach [1] has shown that, unexpectedly, the same is not true for particles orbiting black holes: for Kerr black holes with the spin parameter $a>0.9953$, the velocity has a positive radial gradient for geodesic, stable, circular orbits in a small radial range close to the black hole horizon. We show here that the Aschenbach effect occurs also for non-geodesic circular orbits with constant specific angular momentum $\ell=\ell_{0}=$ const. In Newton's theory it is $\mathcal{V}=\ell_{0} / \mathcal{R}$, with $\mathcal{R}$ being the cylindrical radius. The equivelocity surfaces coincide with the $\mathcal{R}=$ const surfaces which, of course, are just co-axial cylinders. It was previously known that in the black hole case this simple topology changes because one of the "cylinders" self-crosses. We show here that the Aschenbach effect is connected to a second topology change that for the $\ell=$ const tori occurs only for very highly spinning black holes, $a>0.99979$.
\end{abstract}

PACS numbers: 04.20.-q, 04.70.-s, 95.30.-k

\section{INTRODUCTION}

Aschenbach [1] found a very interesting and rather surprising fact about the circular orbits of free particles around the Kerr black holes with high spin. Contrary to what is true for Kerr black holes with a small spin, for orbits around Kerr black holes with $a>0.9953$ the Keplerian orbital velocity $\mathcal{V}_{\mathrm{LNRF}}^{(\varphi)}$ measured in locally nonrotating frames (LNRF) is a non-monotonic function of radius.

In this article we show that there is a corresponding change of behaviour of orbital velocity in the case of nonKeplerian orbits with constant specific angular momentum [8], $\ell(r, \theta)=$ const: for low spin black holes the radial gradient of the orbital velocity, $\partial \mathcal{V}_{\mathrm{LNRF}}^{(\varphi)} / \partial r$, changes its sign once, but for a sufficiently rapidly rotating black holes, it changes the sign three times.

We discuss the geometrical reason for this puzzling behaviour of orbital velocity in terms of the von Zeipel surfaces, defined as the surfaces of constant $\mathcal{R}(r, \theta) \equiv$ $\ell / \mathcal{V}_{\text {LNRF }}^{(\varphi)}$. In Newton's physics (Euclidean geometry), the von Zeipel surfaces have topology of co-axial cylinders $\mathcal{R}=r \sin \theta=$ const. In the black hole geometry, the topology of the von Zeipel surfaces is remarkably different. It was known for a long time that for a non-rotating black hole one of the von Zeipel surfaces self-crosses at the location of the photon orbit [2]. We found that the

\footnotetext{
*Electronic address: Zdenek.Stuchlik@fpf.slu.cz

${ }^{\dagger}$ Electronic address: Petr.Slany@fpf.slu.cz

‡Electronic address: terek@volny.cz

$\S$ Electronic address: marek@fy.chalmers.se
}

Aschenbach effect is due to a second topology change, as the another surface with a cusp together with toroidal surfaces appear. The second change is strictly connected to the first one, but it occurs only for very rapidly rotating black holes.

In Section II we summarize basic relations characterizing the constant specific angular momentum tori. In Section III the orbital velocity relative to the LNRF is given and its properties are determined. In Section IV the notion of von Zeipel radius is introduced and properties of the von Zeipel surfaces are analyzed. In Section D] we present discussion and some concluding remarks.

\section{CONSTANT SPECIFIC ANGULAR MOMENTUM TORI}

In general, stationary and axially symmetric spacetimes with the line element

$$
\mathrm{d} s^{2}=g_{t t} \mathrm{~d} t^{2}+2 g_{t \varphi} \mathrm{d} t \mathrm{~d} \varphi+g_{\varphi \varphi} \mathrm{d} \varphi^{2}+g_{r r} \mathrm{~d} r^{2}+g_{\theta \theta} \mathrm{d} \theta^{2},
$$

the stationary and axisymmetric fluid tori with the stress-energy tensor $T^{\mu \nu}=(\varrho+p) U^{\mu} U^{\nu}+p g^{\mu \nu}$ are characterized by 4 -velocity field

$$
U^{\mu}=\left(U^{t}, 0,0, U^{\varphi}\right)
$$

with $U^{t}=U^{t}(r, \theta), U^{\varphi}=U^{\varphi}(r, \theta)$, and by the distribution of specific angular momentum

$$
\ell=-\frac{U_{\varphi}}{U_{t}} .
$$


The angular velocity of orbiting matter, $\Omega=U^{\varphi} / U^{t}$, is then related to $\ell$ by the formula

$$
\Omega=-\frac{\ell g_{t t}+g_{t \varphi}}{\ell g_{t \varphi}+g_{\varphi \varphi}}
$$

The tori considered here are assumed to have constant specific angular momentum,

$$
\ell=\ell(r, \theta)=\text { const. }
$$

Their structure is determined by equipotential surfaces $W=W(r, \theta)$ defined by [3, 4]

$$
W-W_{\text {in }}=\ln \frac{U_{t}}{\left(U_{t}\right)_{\text {in }}}
$$

with

$$
\left(U_{t}\right)^{2}=\frac{g_{t \varphi}^{2}-g_{t t} g_{\varphi \varphi}}{g_{t t} \ell^{2}+2 g_{t \varphi} \ell+g_{\varphi \varphi}}
$$

the subscript "in" refers to the inner edge of the torus.

The metric components of the Kerr spacetime (with $a>0)$ in the Boyer-Lindquist coordinates are:

$$
\begin{aligned}
g_{t t} & =-\frac{\Delta-a^{2} \sin ^{2} \theta}{\Sigma}, \\
g_{t \varphi} & =-\frac{2 a r \sin ^{2} \theta}{\Sigma} \\
g_{\varphi \varphi} & =\frac{A \sin ^{2} \theta}{\Sigma}
\end{aligned}
$$

where

$$
\begin{aligned}
\Delta & =r^{2}-2 r+a^{2} \\
\Sigma & =r^{2}+a^{2} \cos ^{2} \theta \\
A & =\left(r^{2}+a^{2}\right)^{2}-\Delta a^{2} \sin ^{2} \theta .
\end{aligned}
$$

We make our formulae dimensionless by using the standard $c=G=M=1$ convention. The relation (44) for the angular velocity of matter orbiting the black hole acquires the form

$$
\Omega=\Omega(r, \theta ; a, \ell)=\frac{\left(\Delta-a^{2} \sin ^{2} \theta\right) \ell+2 a r \sin ^{2} \theta}{(A-2 \ell a r) \sin ^{2} \theta}
$$

and the potential W, defined in Eq. (6), has the explicit form

$$
\begin{aligned}
& W=W(r, \theta ; a, \ell)= \\
& \quad \frac{1}{2} \ln \frac{\Sigma \Delta \sin ^{2} \theta}{\left(r^{2}+a^{2}-a \ell\right)^{2} \sin ^{2} \theta-\Delta\left(\ell-a \sin ^{2} \theta\right)^{2}} .
\end{aligned}
$$

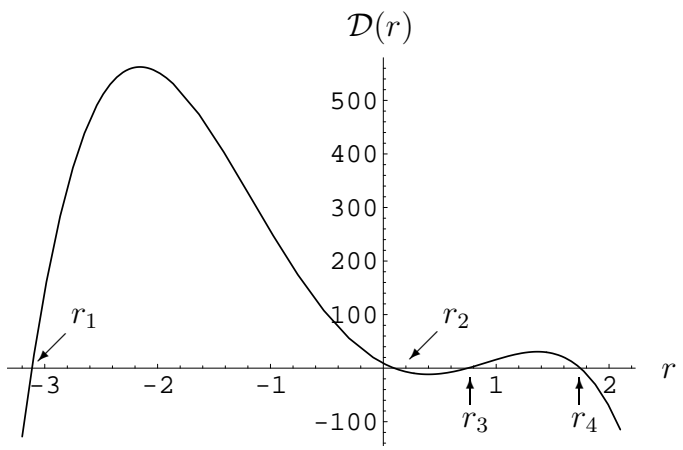

FIG. 1: Reality condition for the existence of local extrema of the function $\ell_{\text {ex }}(r ; a)$. The extrema are allowed, if $\mathcal{D}(r)>0$. Clearly, the physically relevant extrema, located above the outer horizon, can exist in the interval $r \in\left(1, r_{4}\right)$.

\section{THE ORBITAL VELOCITY IN LNRF}

The locally non-rotating frames are given by the tetrad of 1 -forms [, 6 ]

$$
\begin{aligned}
\mathbf{e}^{(t)} & =\left(\frac{\Sigma \Delta}{A}\right)^{1 / 2} \mathbf{d} t, \\
\mathbf{e}^{(r)} & =\left(\frac{\Sigma}{\Delta}\right)^{1 / 2} \mathbf{d} r, \\
\mathbf{e}^{(\theta)} & =\Sigma^{1 / 2} \mathbf{d} \theta, \\
\mathbf{e}^{(\varphi)} & =\left(\frac{A}{\Sigma}\right)^{1 / 2} \sin \theta(\mathbf{d} \varphi-\omega \mathbf{d} t)
\end{aligned}
$$

where the angular velocity of LNRF, $\omega=-g_{t \varphi} / g_{\varphi \varphi}$, is given by the relation

$$
\omega=\frac{2 a r}{A} .
$$

The azimuthal component of 3-velocity in LNRF reads

$$
\mathcal{V}_{\mathrm{LNRF}}^{(\varphi)}=\frac{U^{\mu} \mathrm{e}_{\mu}^{(\varphi)}}{U^{\nu} \mathrm{e}_{\nu}^{(t)}}=\frac{A \sin \theta}{\Sigma \sqrt{\Delta}}(\Omega-\omega) .
$$

Substituting for the angular velocities $\Omega$ and $\omega$ from the relations (14) and (20), respectively, we arrive at the formula

$$
\mathcal{V}_{\mathrm{LNRF}}^{(\varphi)}=\frac{A\left(\Delta-a^{2} \sin ^{2} \theta\right)+4 a^{2} r^{2} \sin ^{2} \theta}{\Sigma \sqrt{\Delta}(A-2 a \ell r) \sin \theta} \ell .
$$
to

In the equatorial plane, $\theta=\pi / 2$, equation (22) reduces

$$
\mathcal{V}^{(\varphi)}(r, \theta=\pi / 2 ; a, \ell)=\frac{r \sqrt{\Delta}}{r\left(r^{2}+a^{2}\right)-2 a(\ell-a)} \ell .
$$

The velocity vanishes at infinity, $r \rightarrow \infty$, and at the horizon, $r \rightarrow r_{+}=1+\sqrt{1-a^{2}}$. Thus, a change of the sign of the radial gradient of velocity occurs for any pair 


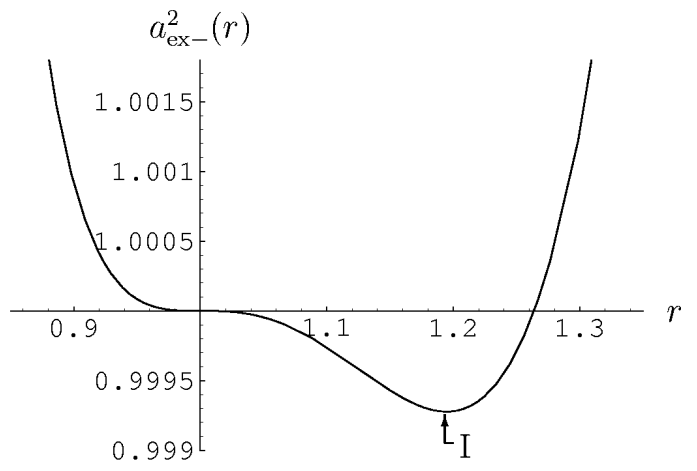

(a)

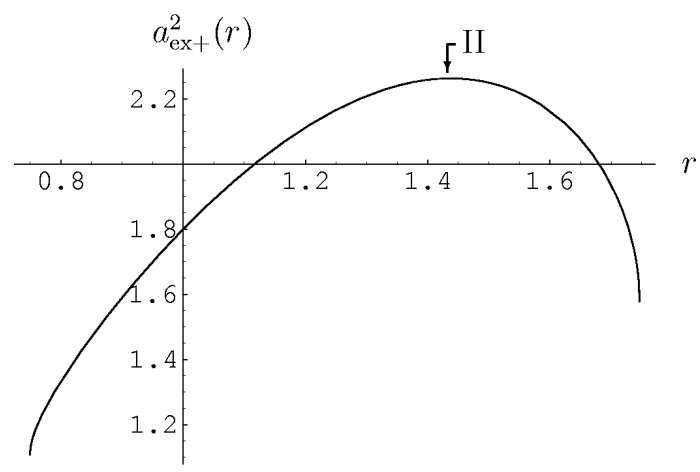

(b)

FIG. 2: Loci of local extrema of the function $\ell_{\mathrm{ex}}(r ; a)$. They are determined by the functions $a_{\mathrm{ex} \pm}^{2}(r)$. (a) The function $a_{\mathrm{ex}-}^{2}(r)$ is relevant for both black holes and naked singularities; its local minimum is denoted I. (b) The function $a_{\mathrm{ex}+}^{2}(r)$ is relevant for naked singularities only; its local maximum is denoted II. $a, \ell$. This is not true for Keplerian orbits. To see this, one may use the formula for Keplerian angular velocity 5]

$$
\Omega=\Omega_{\mathrm{K}}(r ; a)=\frac{1}{\left(r^{3 / 2}+a\right)}
$$

together with equation (21) to get, in the equatorial plane,

$$
\mathcal{V}_{\mathrm{K}}^{(\varphi)}(r ; a)=\frac{\left(r^{2}+a^{2}\right)^{2}-a^{2} \Delta-2 a r\left(r^{3 / 2}+a\right)}{r^{2}\left(r^{3 / 2}+a\right) \sqrt{\Delta}}
$$

Obviously, the velocity formally diverges at $r=r_{+}$. The Keplerian specific angular momentum is given by

$$
\ell_{\mathrm{K}}(r ; a)=\frac{r^{2}-2 a r^{1 / 2}+a^{2}}{r^{3 / 2}-2 r^{1 / 2}+a}
$$

The minimum of $\ell_{\mathrm{K}}(r ; a)$ corresponds to the marginally stable circular geodesic at $r=r_{\mathrm{ms}}$, which is the innermost possible circular, stable geodesic. The innermost possible circular fluid orbit in the constant specific angular momentum tori is given by the condition $\ell=\ell_{\mathrm{K}}\left(r_{\mathrm{in}}\right)$. It is known that $r_{\mathrm{mb}}<r_{\mathrm{in}}<r_{\mathrm{ms}}$. Here $r_{\mathrm{mb}}$ denotes the radius of the circular marginally bound geodesic.

The radial gradient of the equatorial orbital velocity of tori reads

$$
\frac{\partial \mathcal{V}^{(\varphi)}}{\partial r}=\frac{[\Delta+(r-1) r]\left[r\left(r^{2}+a^{2}\right)-2 a(\ell-a)\right]-r\left(3 r^{2}+a^{2}\right) \Delta}{\left[r\left(r^{2}+a^{2}\right)-2 a(\ell-a)\right]^{2} \sqrt{\Delta}} \ell
$$

and it changes its orientation at radii determined by the condition

$$
\ell=\ell_{\mathrm{ex}}(r ; a) \equiv a+\frac{r^{2}\left[\left(r^{2}+a^{2}\right)(r-1)-2 r \Delta\right]}{2 a[\Delta+r(r-1)]}
$$

We have to discuss properties of $\ell_{\mathrm{ex}}(r ; a)$ above the event horizon $r_{+}$taking into account the limits on the inner boundary of the tori, $\ell \in\left(l_{\mathrm{ms}}, l_{\mathrm{mb}}\right)$ where $\ell_{\mathrm{ms}}\left(\ell_{\mathrm{mb}}\right)$ denotes specific angular momentum of the marginally stable (marginally bound) circular geodesic. The local extrema of $\ell_{\mathrm{ex}}(r ; a)$ are given by the relation

$$
a^{2}=a_{\mathrm{ex} \pm}^{2}(r) \equiv r \frac{3+18 r-7 r^{2} \pm \sqrt{\mathcal{D}(r)}}{2(3 r+2)}
$$

with

$$
\begin{gathered}
\mathcal{D}(r)=9-108 r+150 r^{2}-12 r^{3}-23 r^{4}= \\
=-23\left(r-r_{1}\right)\left(r-r_{2}\right)\left(r-r_{3}\right)\left(r-r_{4}\right),
\end{gathered}
$$

where

$$
\begin{aligned}
& r_{1} \doteq-3.11363 \\
& r_{2} \doteq 0.09602, \\
& r_{3} \doteq 0.74939 \\
& r_{4} \doteq 1.74648 .
\end{aligned}
$$

The situation is illustrated in Fig. 11 which implies that only the interval $r \in\left(r_{3}, r_{4}\right)$ is relevant for the region outside of the black-hole event horizon. Behaviour of the functions $a_{\mathrm{ex} \pm}^{2}(r)$ is given in Fig. 2 Clearly, only $a_{\mathrm{ex}-}^{2}(r)$ 


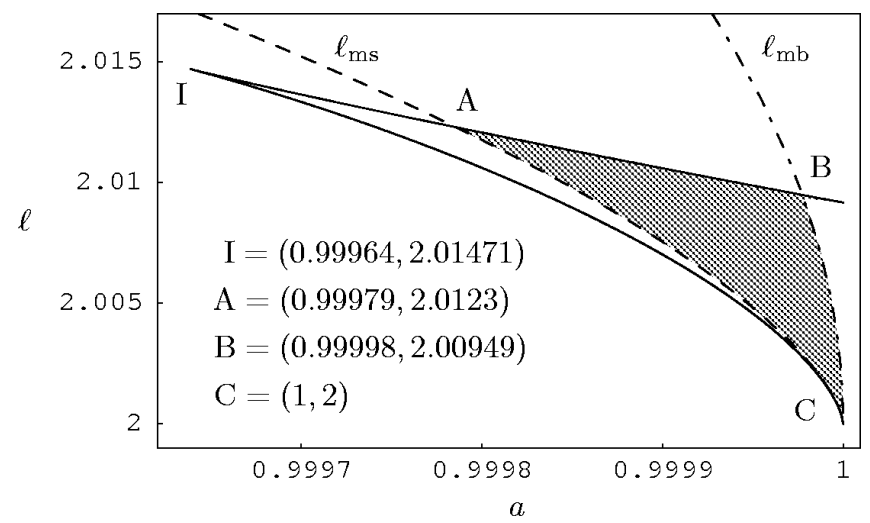

FIG. 3: Kerr spacetimes with the change of sign of the gradient of LNRF velocity. In the $\ell-a$ plane, the functions $\ell_{\mathrm{ex}(\max )}(a)$ (upper solid curve), $\ell_{\mathrm{ex}(\min )}(a)$ (lower solid curve), $\ell_{\mathrm{ms}}(a)$ (dashed curve) and $\ell_{\mathrm{mb}}(a)$ (dashed-dotted curve) are given. For pairs of $(a, \ell)$ from the shaded region, the gradient of the orbital velocity of tori changes its sign twice inside the tori. Between the points $\mathrm{A}, \mathrm{B}$, the $\ell_{\operatorname{ex}(\max )}(a)$ curve determines an inflex point of $\mathcal{V}^{(\varphi)}(r ; a, \ell)$. The inflex points determined by the curve $\ell_{\operatorname{ex}(\min )}(a)$ are irrelevant being outside of the definition region for constant specific angular momentum tori, $\ell \in\left(\ell_{\mathrm{ms}}(a), \ell_{\mathrm{mb}}(a)\right)$. The point I corresponds to the inflex point of $\ell_{\mathrm{ex}}(r ; a)$; cf. Fig. [2].

is relevant for black holes. The minimum of $a_{\mathrm{ex}-}^{2}(r)$, denoted $\mathrm{I}$, is located at radius $r_{\text {min }} \doteq 1.19466$ and the critical value of the rotational parameter is

$$
a_{\mathrm{c}(\mathrm{bh})} \doteq \sqrt{0.99928} \doteq 0.99964
$$

Note that both the functions $a_{\mathrm{ex} \pm}^{2}(r)$ are relevant for Kerr naked singularities. The maximum of $a_{\mathrm{ex}+}^{2}(r)$, denoted II, is located at radius $r_{\max } \doteq 1.43787$ and the critical value of the rotational parameter is

$$
a_{\mathrm{c}(\mathrm{ns})} \doteq \sqrt{2.26289} \doteq 1.50429 .
$$

Therefore, the possibility to have three changes of the sign of $\partial \mathcal{V}^{(\varphi)} / \partial r$ in constant specific angular momentum tori is limited from bellow for black holes, and from above for naked singularities. Here we restrict our attention to the Kerr black holes.

Now, we have to compare the local extrema of the function $\ell_{\mathrm{ex}}(r ; a)$, determined by the condition (29), with the functions characterizing the marginally stable, $\ell_{\mathrm{ms}}(a)$, and the marginally bound, $\ell_{\mathrm{mb}}(a)$, circular geodesics as these determine the limits of allowance of stationary toroidal structures in the Kerr spacetimes [3]. For each given value of $a$, location of both the marginally stable and the marginally bound circular geodesics is uniquely given by the functions $r_{\mathrm{ms}}=r_{\mathrm{ms}}(a), r_{\mathrm{mb}}=$ $r_{\mathrm{mb}}(a)$ [5], and $\ell_{\mathrm{ms}}(a), \ell_{\mathrm{mb}}(a)$ can then be determined using the formulae for $\ell_{\mathrm{K}}(r ; a)$ and $r_{\mathrm{ms}}(a), r_{\mathrm{mb}}(a)$, respectively. In Fig. 3 behaviour of the local extrema $\ell_{\mathrm{ex}(\min )}(a), \ell_{\mathrm{ex}(\max )}(a)$ and the functions $\ell_{\mathrm{ms}}(a), \ell_{\mathrm{mb}}(a)$ is illustrated. It is clear immediately that the sign's change of $\partial \mathcal{V}^{(\varphi)} / \partial r$ is relevant only for tori orbiting the Kerr black holes with the rotational parameter

$$
a>a_{\mathrm{c}(\text { tori })} \doteq 0.99979,
$$

which is much higher than the critical value $a_{\mathrm{c}(\mathrm{K})} \doteq$ 0.9953 determined by Aschenbach for Keplerian discs [1]. For $a>a_{\mathrm{c}(\mathrm{tori})}$ the relevance of $\ell_{\mathrm{ex}}(r ; a)$ is limited from bellow by $\ell_{\mathrm{ms}}(a)$. There is another critical value of the rotational parameter, $a=a_{\mathrm{c}(\mathrm{mb})} \doteq 0.99998$, where $\ell_{\mathrm{mb}}(a)=\ell_{\mathrm{ex}(\max )}(a)$; for $a>a_{\mathrm{c}(\mathrm{mb})}$ the relevance of $\ell_{\mathrm{ex}}(r ; a)$ is limited from above by $\ell_{\mathrm{mb}}(a)$.

The character of the region, where $\partial \mathcal{V}^{(\varphi)} / \partial r$ changes sign, can be properly illustrated by considering the functions $\ell_{\mathrm{ex}}(r ; a)$ and $\ell_{\mathrm{K}}(r ; a)$ simultaneously. First, we show that there is no common point of those functions in black-hole spacetimes with $a<1$. Indeed, the condition $\ell_{\mathrm{ex}}(r ; a)=\ell_{\mathrm{K}}(r ; a)$ implies an equation quartic in $a$, which has four solutions

$$
\begin{aligned}
a & =a_{1}(r) \equiv r \sqrt{r} \\
a & =a_{2}(r) \equiv-\sqrt{r(2-r)}, \\
a & =a_{\mathrm{h}}(r) \equiv \sqrt{r(2-r)}, \\
a & =a_{\mathrm{ph}+}(r) \equiv \frac{\sqrt{r}}{2}(3-r) .
\end{aligned}
$$

The solution $a_{1}(r)>1$ at $r>1$, i.e., it corresponds to naked singularities at $r>1$, the solution $a_{2}(r)$ is negative everywhere, the solution $a_{3}=a_{\mathrm{h}}(r)$ determines radius of the event horizon, while the solution $a_{4}=a_{\mathrm{ph}+}(r)$ determines radius of the corotating photon circular geodesic. None of the solutions is relevant for the stationary tori. We can conclude that above the photon circular orbit there is always $\ell_{\mathrm{K}}(r ; a)>\ell_{\mathrm{ex}}(r ; a)$; therefore, the innermost local maximum of $\mathcal{V}^{(\varphi)}(r ; a)$ for $a>a_{\mathrm{c}(\mathrm{bh})}$, and the only local maximum of $\mathcal{V}^{(\varphi)}(r ; a)$ for $a<a_{\mathrm{c}(\mathrm{bh})}$, is always physically irrelevant in constant specific angular momentum tori.

For black-hole spacetimes, behaviour of the functions $\ell_{\mathrm{ex}}(r ; a)$ and $\ell_{\mathrm{K}}(r ; a)$ can then be classified into six classes which are illustrated in Fig. [4.

1. $0<a<a_{\mathrm{c}(\mathrm{bh})}$ : No extrema of $\ell_{\mathrm{ex}}(r ; a)$ (Fig. 固).

2. $a=a_{\mathrm{c}(\mathrm{bh})}$ : An inflex point of $\ell_{\mathrm{ex}}(r ; a)$ (Fig. 目).

3. $a_{\mathrm{c}(\mathrm{bh})}<a<a_{\mathrm{c}(\mathrm{tori})}$ : Two local extrema of $\ell_{\mathrm{ex}}(r ; a)$ present, but out of the region allowing the existence of tori (Fig. 迎).

4. $a_{\mathrm{c}(\text { tori })}<a<a_{\mathrm{c}(\mathrm{mb})}$ : Two local extrema of $\ell_{\mathrm{ex}}(r ; a)$ allowed in the region of $\ell \in\left(\ell_{\mathrm{ms}}, \ell_{\mathrm{ex}(\max )}\right)$ (Fig. 4 $\left.\mathrm{d}\right)$.

5. $a_{\mathrm{c}(\mathrm{mb})}<a<1$ : Two local extrema of $\ell_{\mathrm{ex}}(r ; a)$ allowed in the region $\ell \in\left(\ell_{\mathrm{ms}}, \ell_{\mathrm{mb}}\right)$ (Fig. 目).

6. $a=1$ : The minimum of $\ell_{\mathrm{ex}}(r ; a)$ coincides with the marginally bound geodesic with $\ell_{\mathrm{mb}}=2$ at $r_{\mathrm{mb}}=1$. The curves $\ell_{\mathrm{ex}}(r ; a=1)$ and $\ell_{\mathrm{K}}(r ; a=1)$ intersect at $r=1$ (Fig. 目). 


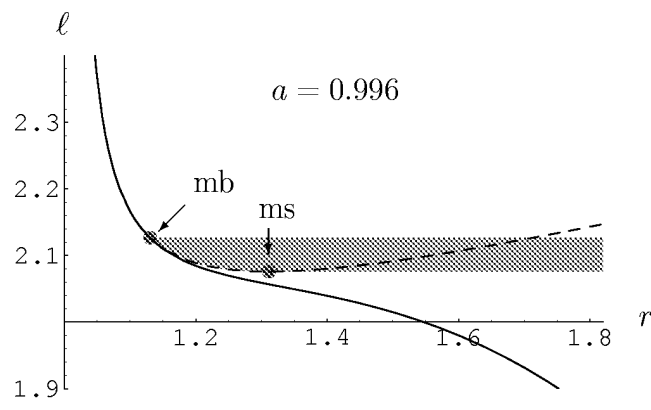

(a)

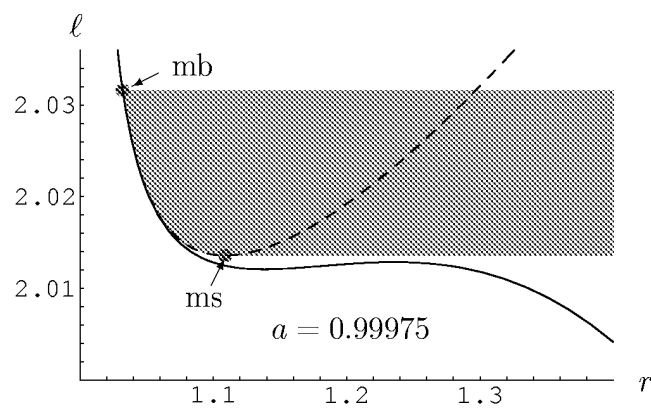

(c)

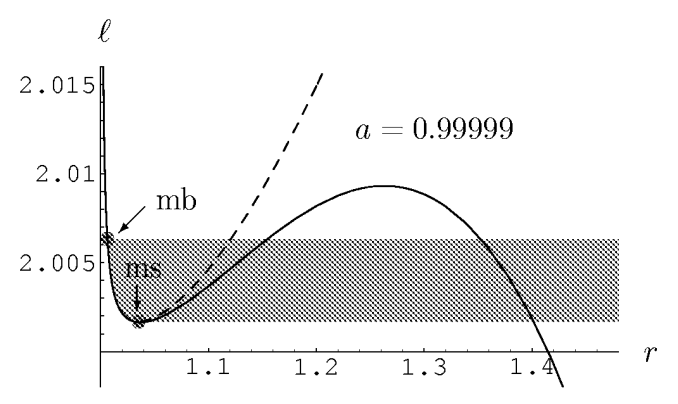

(e)

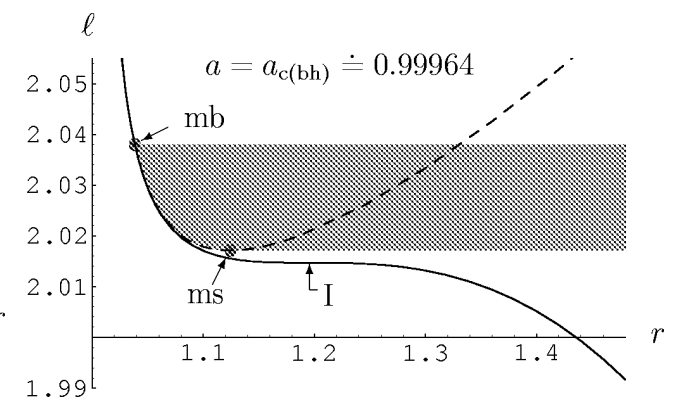

(b)

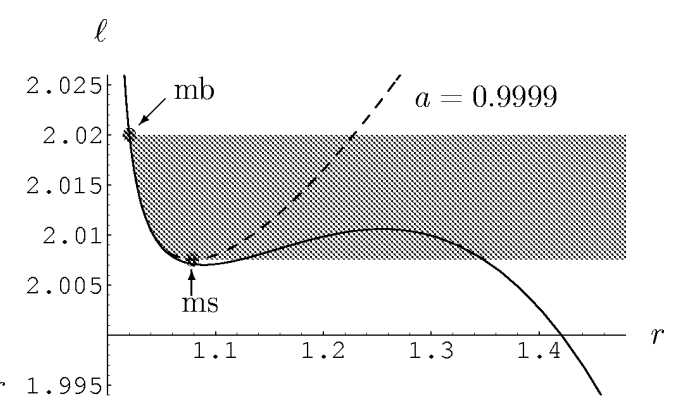

(d)

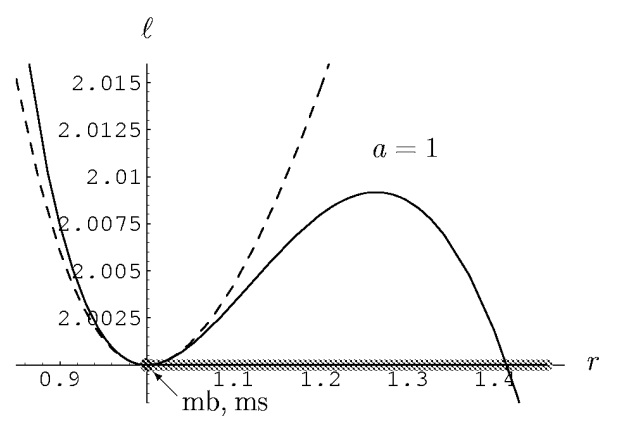

(f)

FIG. 4: Classification of the Kerr black-hole spacetimes according to the properties of the functions $\ell_{\mathrm{ex}}(r ; a)$ (solid curves) and $\ell_{\mathrm{K}}(r ; a)$ (dashed curves). The functions are plotted for six cases corresponding to the classification. The constant specific angular momentum tori can exist in the shaded region only along $\ell=$ const lines. Their inner edge (center) is determined by the decreasing (increasing) part of $\ell_{\mathrm{K}}(r ; a)$. The local extrema of the orbital velocity relative to LNRF relevant for tori are given by the intersections of $\ell=$ const line with the curve of $\ell_{\mathrm{ex}}(r ; a)$ in the shaded region. Notice that the region corresponding to the allowed values of $\ell$ for the discs is narrowing with $a \rightarrow 1$, it is degenerated into the $\ell=2$ line for $a=1$ as $\ell_{\mathrm{ms}}=\ell_{\mathrm{mb}}=2$ in this case. In the case (e), the gradient $\partial \mathcal{V}^{(\varphi)} / \partial r$ changes sign for all values of $\ell \in\left(\ell_{\mathrm{ms}}, \ell_{\mathrm{mb}}\right)$ allowed for the tori, while in the case (d), it is allowed for a region restricted from above by the value $\ell_{\operatorname{ex}(\max )}(a)$. In the cases (a)-(c), the change of sign of $\partial \mathcal{V}^{(\varphi)} / \partial r$ cannot occur in the disc. It is directly seen from cases (d)-(f) that the gradient $\partial \mathcal{V}^{(\varphi)} / \partial r$ changes the sign closely above the center of the disc.

Clearly, three changes of sign of $\partial \mathcal{V}^{(\varphi)} / \partial r$ can occur for Kerr black holes with the rotational parameter $a>$ $a_{\mathrm{c}(\mathrm{bh})} \doteq 0.99964$. However, the effect is relevant for constant specific angular momentum tori only if $a>$ $a_{\mathrm{c} \text { (tori) }} \doteq 0.99979$. The interval of corresponding values of the specific angular momentum $\ell \in\left(\ell_{\mathrm{ms}}(a), \ell_{\mathrm{ex}(\max )}(a)\right)$ grows with $a$ growing up to the critical value of $a_{\mathrm{c}(\mathrm{mb})} \doteq$ 0.99998. For $a>a_{\mathrm{c}(\mathrm{mb})}$, the interval of relevant values of $\ell \in\left(\ell_{\mathrm{ms}}(a), \ell_{\mathrm{mb}}(a)\right)$ is narrowing with growing of the rotational parameter up to $a=1$, which corresponds to a singular case where $\ell_{\mathrm{ms}}(a=1)=\ell_{\mathrm{mb}}(a=1)=2$. Notice that the situation becomes to be singular only in terms of the specific angular momentum; it is shown [5] that for $a=1$ both the total energy $\mathcal{E}$ and the axial angular momentum $\mathcal{L}$ differ at $r_{\mathrm{ms}}$ and $r_{\mathrm{mb}}$, respectively, but their combination, $\ell \equiv \mathcal{L} / \mathcal{E}$, giving the specific angular momentum, coincides at these radii. 


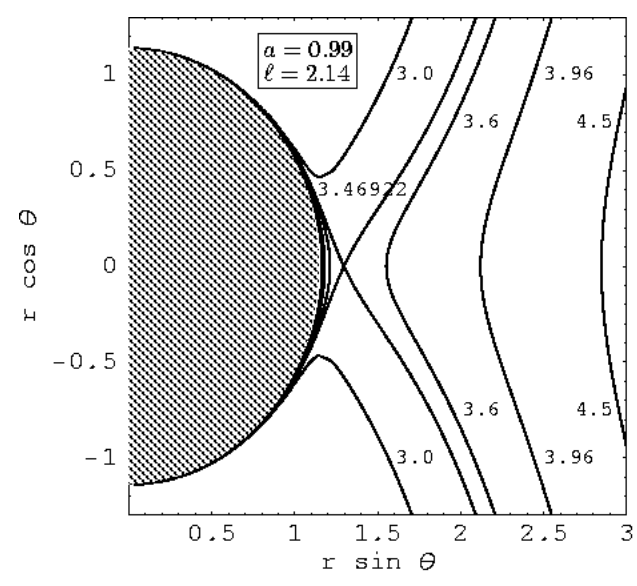

(a)

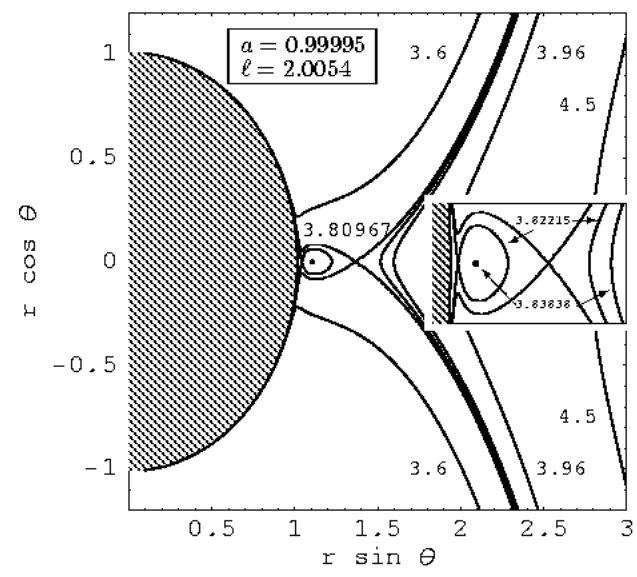

(c)

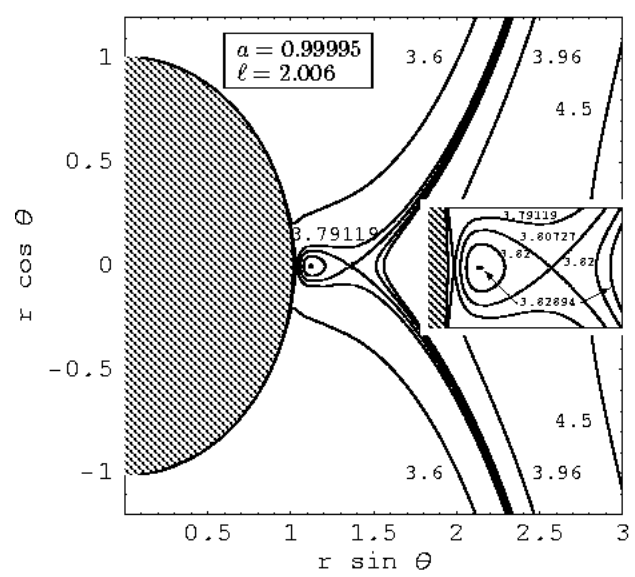

(e)

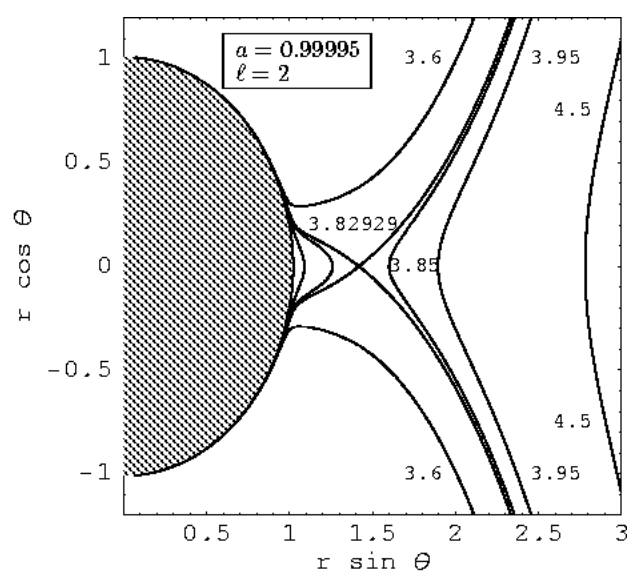

(b)

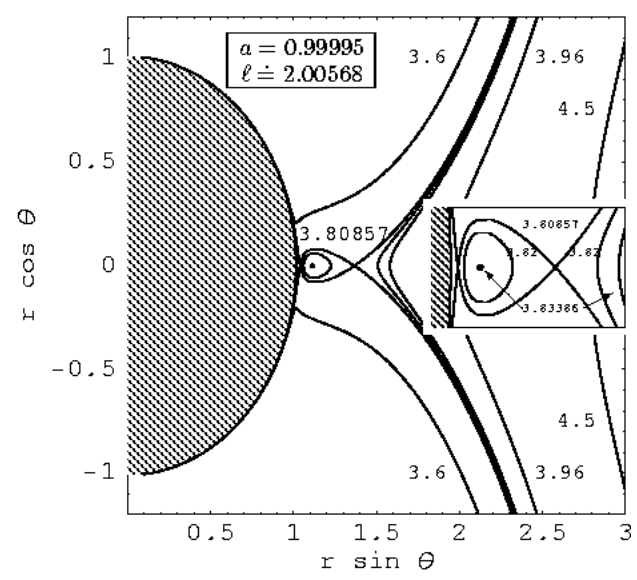

(d)

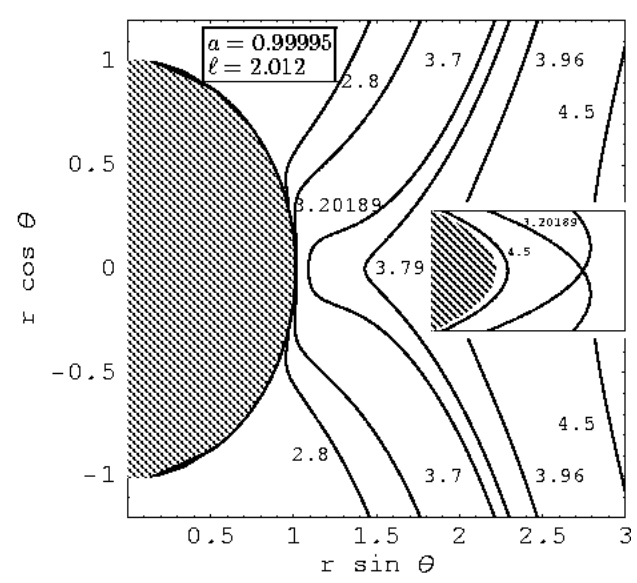

(f)

FIG. 5: Von Zeipel surfaces (meridional sections). (a) For $a<a_{\mathrm{c}(\mathrm{bh})}$ and any $\ell$, only one surface with a cusp in the equatorial plane and no closed (toroidal) surfaces exist. The cusp is, however, located outside the toroidal equilibrium configurations of perfect fluid. (b)-(f) For $a>a_{\mathrm{c}(\mathrm{bh})}$ and $\ell$ appropriately chosen, two surfaces with a cusp ((c), (e)), or one surface with both the cusps (d), together with closed (toroidal) surfaces, exist. Moreover, if $a>a_{\mathrm{c}(\text { tori })}$, both the outer cusp and the central ring of closed surfaces are located inside the toroidal equilibrium configurations corresponding to constant specific angular momentum discs (cases (c)-(e)). If $\ell$ is sufficiently low/high $((\mathrm{b}) /(\mathrm{f}))$, there is only one surface with a cusp outside the configuration. Shaded region corresponds to the black hole. 


\section{VON ZEIPEL SURFACES}

Till now, our study was focused on the properties of LNRF velocity profile in the equatorial plane of the constant specific angular momentum tori. However, it is useful to obtain global characteristics of the phenomenon that is shown to be manifested in the equatorial plane as the existence of a small region with positive gradient of the LNRF velocity.

It is well known that rotational properties of perfect fluid equilibrium configurations in strong gravity are well represented by the radius of gyration $\widetilde{\mathcal{R}}$, introduced in the case of spherically symmetric Schwarzschild spacetimes in [2], as the direction of increase of $\widetilde{\mathcal{R}}$ defines a local outward direction of the dynamical effects of rotation of the fluid. In the stationary and axisymmetric spacetimes, the radius of gyration was defined by the relation []

$$
\widetilde{\mathcal{R}} \equiv\left(\frac{\tilde{\ell}}{\widetilde{\Omega}}\right)^{1 / 2}
$$

where $\widetilde{\Omega}=\Omega-\omega$ is the angular velocity relative to the LNRF. However, $\tilde{\ell} \equiv \mathcal{L} / \widetilde{\mathcal{E}}$ is not the specific angular momentum $\ell \equiv \mathcal{L} / \mathcal{E}$ with $\mathcal{L}=p_{\mu} \xi_{(\varphi)}^{\mu}, \mathcal{E}=-p_{\mu} \xi_{(t)}^{\mu}$ being the 4-momentum projections on the Killing vector fields $\xi_{(\varphi)}^{\mu}=\delta_{\varphi}^{\mu}$ and $\xi_{(t)}^{\mu}=\delta_{t}^{\mu}$, but $\widetilde{\mathcal{E}}=-p_{\mu} \tilde{\eta}^{\mu}$, where $\tilde{\eta}^{\mu}=$ $\xi_{(t)}^{\mu}+\omega \xi_{(\varphi)}^{\mu}$ is not a Killing vector field, i.e., $\widetilde{\mathcal{E}}$ is related to the LNRF and it is not a constant of motion. Important consequence of such a definition is given by the relation between $\widetilde{\mathcal{R}}$ and $\mathcal{V}_{\mathrm{LNRF}}^{(\varphi)}$ :

$$
\mathcal{V}_{\mathrm{LNRF}}^{(\varphi)}=\widetilde{\Omega} \widetilde{\mathcal{R}}
$$

Here, we shall use another physically reasonable way of defining a global quantity characterizing rotating fluid configurations by using directly the LNRF orbital velocity. We define, so-called, von Zeipel radius by the relation

$$
\mathcal{R} \equiv \frac{\ell}{\mathcal{V}_{\text {LNRF }}^{(\varphi)}}
$$

which generalizes the Schwarzschildian definition of gyration radius. In static spacetimes, the von Zeipel radius (40) coincides with the radius of gyration defined by the relation (38), however, in stationary, axisymmetric spacetimes, relation between the both radii has the form

$$
\mathcal{R}=(1-\omega \ell) \widetilde{\mathcal{R}}
$$

In the case of tori with $\ell(r, \theta)=$ const, the von Zeipel surfaces, i.e., the surfaces of $\mathcal{R}(r, \theta ; a, \ell)=$ const, coincide with the equivelocity surfaces $\mathcal{V}_{\mathrm{LNRF}}^{(\varphi)}(r, \theta ; a, \ell)=$ const. For the tori in the Kerr spacetimes, there is

$$
\mathcal{R}(r, \theta ; a, \ell)=\frac{\Sigma \sqrt{\Delta}(A-2 a \ell r) \sin \theta}{A\left(\Delta-a^{2} \sin ^{2} \theta\right)+4 a^{2} r^{2} \sin ^{2} \theta} .
$$

Topology of the von Zeipel surfaces can be directly determined by the behaviour of the von Zeipel radius (42) in the equatorial plane

$$
\mathcal{R}(r, \theta=\pi / 2 ; a, \ell)=\frac{r\left(r^{2}+a^{2}\right)-2 a(\ell-a)}{r \sqrt{\Delta}} .
$$

The local minima of the function (43) determine loci of the cusps of the von Zeipel surfaces, while its local maximum (if it exists) determines a circle around which closed toroidally shaped von Zeipel surfaces are concentrated (Fig. 5). Notice that the minima (maximum) of $\mathcal{R}(r, \theta=\pi / 2 ; a, \ell)$ correspond(s) to the maxima (minimum) of $\mathcal{V}_{\mathrm{LNRF}}^{(\varphi)}(r, \theta=\pi / 2 ; a, \ell)$, therefore, the inner cusp is always physically irrelevant being located outside of the toroidal configuration of perfect fluid, cf. Fig. 4

\section{DISCUSSION AND CONCLUSIONS}

It is useful to discuss both the qualitative and quantitative aspects of the phenomenon of the positive gradient of LNRF orbital velocity. In the Kerr spacetimes with $a>a_{\mathrm{c}(\text { tori })}$, changes of sign of the gradient of $\mathcal{V}^{(\varphi)}(r ; a, \ell)$ must occur closely above the center of relevant toroidal discs, at radii corresponding to stable circular geodesics of the spacetime (cf. Fig. 4).

For $a=a_{\mathrm{c}(\text { tori })} \doteq 0.99979$, except the irrelevant local maximum located always outside the disc, an inflex point of $\mathcal{V}^{(\varphi)}(r ; a, \ell)$ occurs at $r_{\text {inf }} \doteq 1.24143$ for the disc with $\ell=\ell_{\mathrm{ms}} \doteq 2.0123$. With rotational parameter growing $\left(a>a_{\mathrm{c}(\text { tori })}\right)$, the local maximum of $\mathcal{V}^{(\varphi)}(r ; a, \ell)$ is succesively shifted up to values of $r \sim 1.4$, while the local minimum of $\mathcal{V}^{(\varphi)}(r ; a, \ell)$ is shifted down to $r=1$ in the limit of $a=1$ (Fig. 7a). Notice that the function $\mathcal{V}^{(\varphi)}(r ; a, \ell)$ can possess two local maxima even for $a>a_{\mathrm{c}(\mathrm{bh})} \doteq 0.99964$ but for irrelevant values of the parameter $\ell$, as it does not enters the interval corresponding to the constant specific angular momentum tori, $\ell \notin\left(\ell_{\mathrm{ms}}, \ell_{\mathrm{mb}}\right)$. The loci of these extreme points can be directly inferred from Fig. 4 where the regions corresponding to the constant specific angular momentum tori are shaded.

For some representative cases corresponding to the classification of Kerr spacetimes given in Sec. [II] behaviour of $\mathcal{V}^{(\varphi)}(r ; a, \ell)$ is illustrated in Fig. 6] which enables us to make some conclusions on the quantitative properties of the orbital velocity and its gradient. For comparison, profiles of the Keplerian velocity $\mathcal{V}_{\mathrm{K}}^{(\varphi)}(r ; a)$ are included. With $a$ growing in the region of $a \in\left(a_{\mathrm{c}(\text { tori })}, 1\right)$, the difference $\Delta \mathcal{V}^{(\varphi)} \equiv \mathcal{V}_{\max }^{(\varphi)}-\mathcal{V}_{\min }^{(\varphi)}$ grows as well as the difference of radii, $\Delta r \equiv r_{\max }-r_{\min }$, where the local extrema of $\mathcal{V}^{(\varphi)}(r ; a, \ell)$ occur, see Figs. 7], b. Recall that the innermost local maximum of $\mathcal{V}^{(\varphi)}(r ; a, \ell)$ must be located, necessarilly, under the disc structure. The value of $\mathcal{V}^{(\varphi)}\left(r=r_{\text {in }} ; a, \ell\right)$ at the inner edge of the toroid (where $\ell=\ell_{\mathrm{K}}\left(r_{\mathrm{in}} ; a\right)$ ) is located closer and closer to the local minimum of $\mathcal{V}^{(\varphi)}(r ; a, \ell)$ when $a \rightarrow 1$. For 


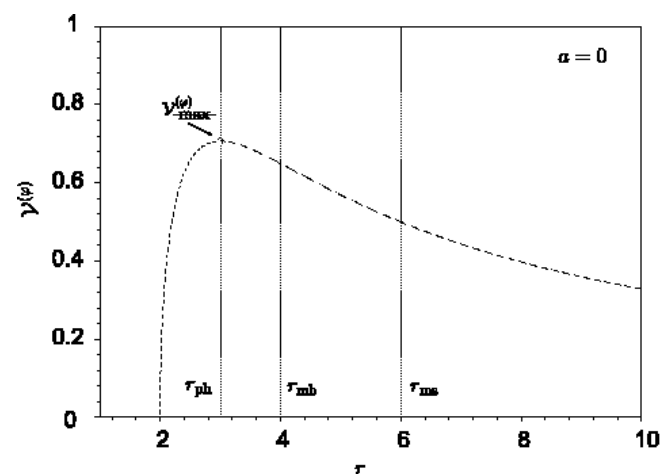

(a)

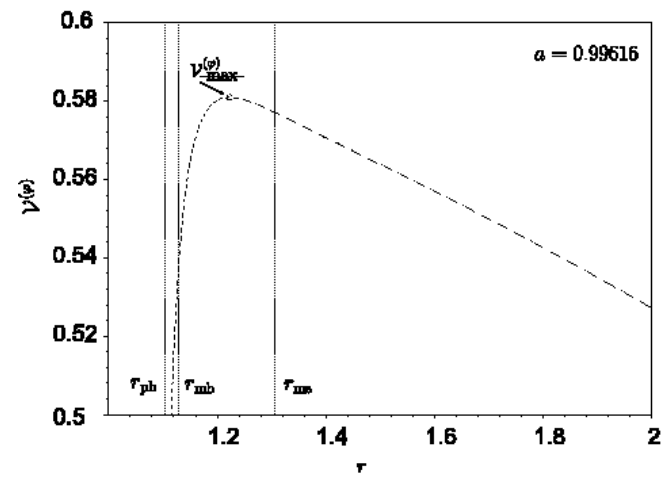

(c)

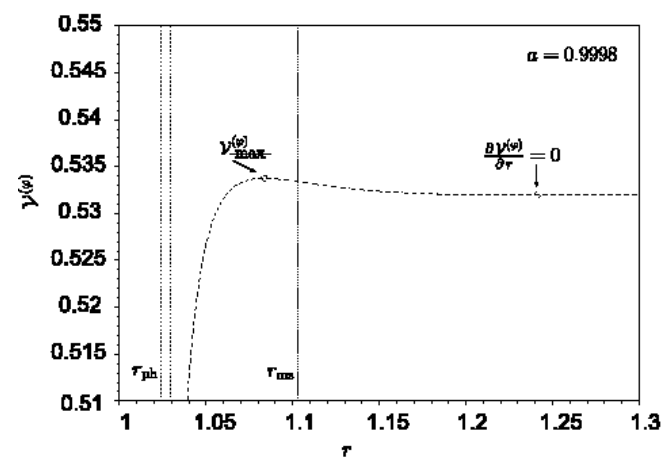

(e)

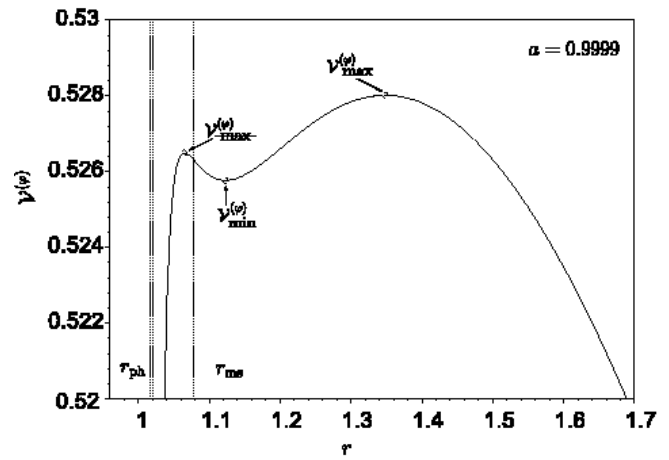

(g)

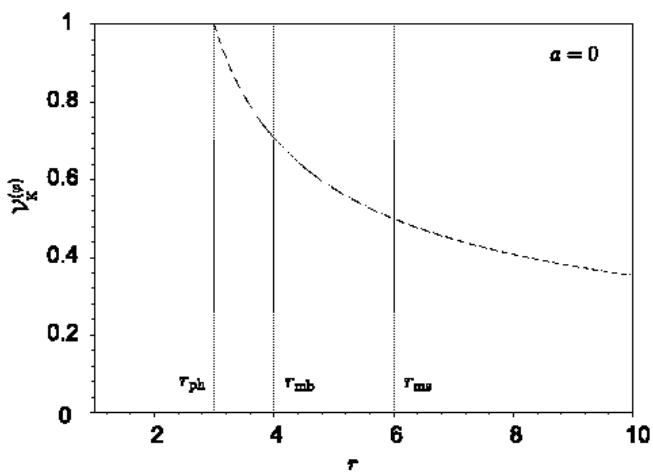

(b)

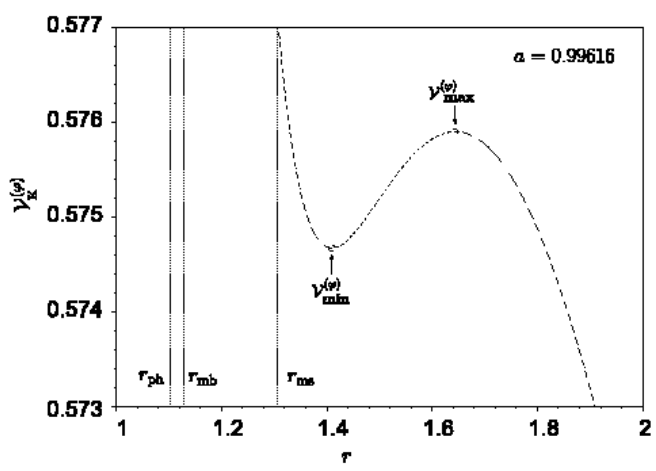

(d)

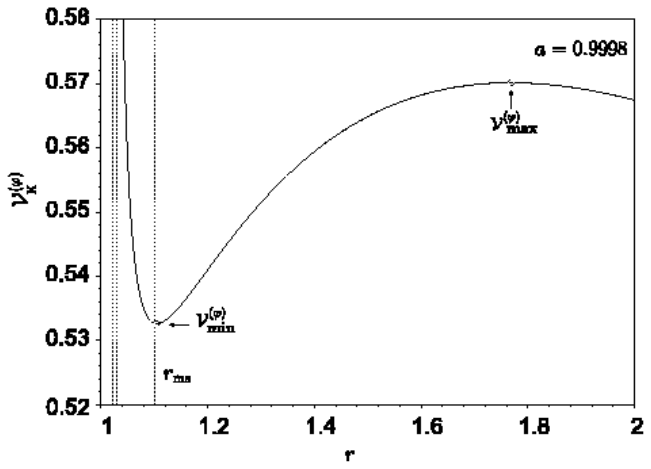

(f)

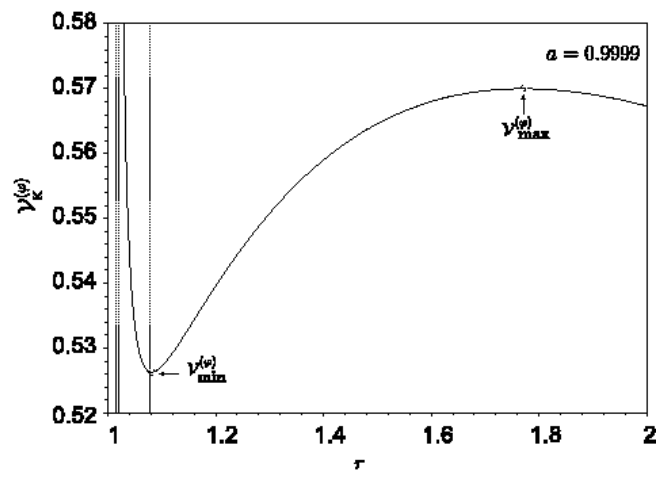

(1)

FIG. 6: Profiles of the equatorial orbital velocity of constant specific angular momentum tori in LNRF in terms of the radial Boyer-Lindquist coordinate. The profiles are given for typical values of $a$ corresponding to the classification of the Kerr blackhole spacetimes. For comparison, the profiles are given for the orbital velocity of Keplerian discs in Kerr spacetimes with the same rotational parameter $a$. For tori, values of $\ell=$ const are appropriately chosen; commonly, $\ell=\ell_{\mathrm{ms}}$ is used giving the maximal value of the velocity difference in between the local extrema, and representing the limiting case of constant specific angular momentum tori. 


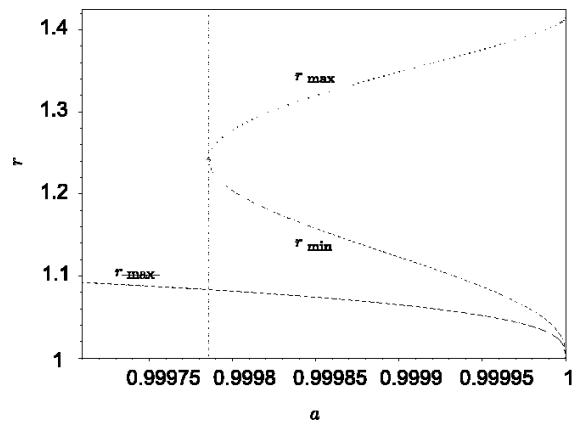

(a)

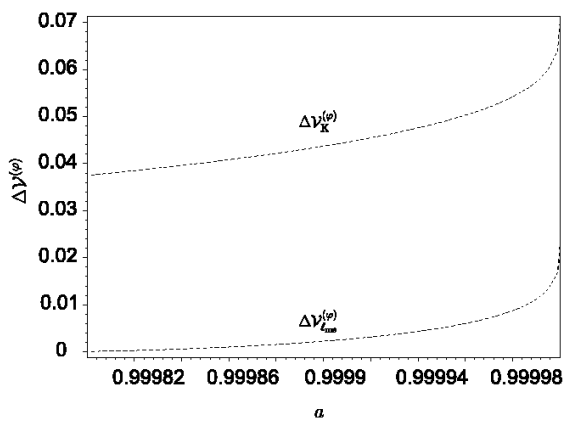

(b)

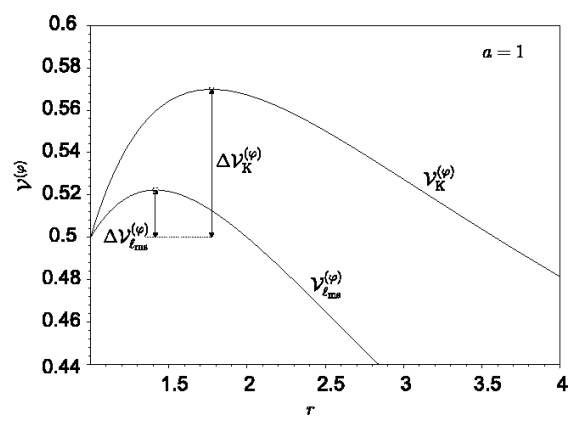

(c)

FIG. 7: (a) Positions of local extrema of $\mathcal{V}_{\mathrm{LNRF}}^{(\varphi)}$ (in B-L coordinates) for the constant specific angular momentum tori with $\ell=\ell_{\mathrm{ms}}$ in dependence on the rotational parameter $a$ of the black hole. (b) Velocity difference $\Delta \mathcal{V}^{(\varphi)}=\mathcal{V}_{\text {max }}^{(\varphi)}-\mathcal{V}_{\text {min }}^{(\varphi)}$ as a function of the rotational parameter $a$ of the black hole for both the Keplerian disc and the constant specific angular momentum (nonKeplerian) disc with $\ell=\ell_{\text {ms }}$. (c) Orbital-velocity curves in the limiting case of the extreme black hole. At $r=1$, the Keplerian orbital velocity $\mathcal{V}_{\mathrm{K}}^{(\varphi)}$ has a local minimum, whereas the orbital velocity $\mathcal{V}_{\ell_{\mathrm{ms}}^{(\varphi)}}$ of the constant specific angular momentum disc has an inflex point. In both cases, the velocity difference $\Delta \mathcal{V}^{(\varphi)}$ reaches its maximal values: $\Delta \mathcal{V}_{\mathrm{K}}^{(\varphi)} \doteq 0.06986, \Delta \mathcal{V}_{\ell_{\mathrm{ms}}}^{(\varphi)} \doteq 0.02241$.

$a=1$, there is an inflex point of $\mathcal{V}^{(\varphi)}(r ; a=1, \ell)$ at $r=1$ where the local minimum and the "forbidden" local maximum of $\mathcal{V}^{(\varphi)}(r ; a, \ell)$ for $\ell=2$ coincide, Fig. [7. Moreover, the velocity difference $\Delta \mathcal{V}^{(\varphi)}$ is smaller but comparable in the tori in comparison with Keplerian discs. We can see that for $a \rightarrow 1$, the velocity difference in the tori $\Delta \mathcal{V}_{\text {tori }}^{(\varphi)} \approx 0.02$, while for the Keplerian discs it goes even up to $\Delta \mathcal{V}_{\mathrm{K}}^{(\varphi)} \approx 0.07$, see Fig. [7. These are really huge velocity differences, being expressed in units of $c$.

We can conclude that the Aschenbach effect, i.e., the change of sign of gradient of the LNRF orbital velocity, in the case of constant specific angular momentum tori occur for the discs orbiting Kerr black holes with the rotational parameter $a>a_{\mathrm{c}(\text { tori })}$. In terms of the redefined rotational parameter, $1-a$, its value of $1-a_{\mathrm{c} \text { (tori) }} \doteq 2.1 \times 10^{-4}$ is more than one order lower than the value $1-a_{\mathrm{c}(\mathrm{K})} \doteq 4.7 \times 10^{-3}$ found by Aschenbach for the changes of sign of the gradient of orbital velocity in Keplerian discs. In constant specific angular momentum tori, the Aschenbach effect is elucidated by topology changes of the von Zeipel surfaces. In addition to one self-crossing von Zeipel surface existing for all values of the rotational parameter $a$, for $a>a_{\mathrm{c}(\text { tori) }}$ the second self-crossing surface together with toroidal surfaces occur. Toroidal von Zeipel surfaces exist under the newly developing cusp, being centered around the circle corresponding to the minimum of the equatorial LNRF velocity profile.

\section{Acknowledgments}

The authors were supported by the Czech GAČR grants 202/02/0735 and 205/03/H144. The main parts of the work were done at the Department of Astrophysics of Chalmers University at Göteborg and at Nordita at Copenhagen. The authors Z.S., P.S. and G.T. would like to express their gratitude to the staff of the Chalmers University and Nordita for perfect hospitality.
[1] B. Aschenbach, Astronomy and Astrophysics 425, 1075 (2004).

[2] M. A. Abramowicz, J. C. Miller, and Z. Stuchlík, Phys. Rev. D 47, 1440 (1993).

[3] M. A. Abramowicz, M. Jaroszyński, and M. Sikora, Astronomy and Astrophysics 63, 221 (1978).

[4] M. Kozłowski, M. Jaroszyński, and M. A. Abramowicz, Astronomy and Astrophysics 63, 209 (1978).

[5] J. M. Bardeen, W. H. Press, and S. A. Teukolsky, Astro- phys. J. 178, 347 (1972).

[6] C. W. Misner, K. S. Thorne, and J. A. Wheeler, Gravitation (Freeman, San Francisco, 1973).

[7] M. A. Abramowicz, P. Nurowski, and N. Wex, Classical Quantum Gravity 12, 1467 (1995).

[8] Here the specific angular momentum means the axial (conserved) angular momentum per total (conserved) energy and we use this term for both particles and fluid elements in the whole article. 